\title{
Ion Implantation into Presolar Grains: A Theoretical Model
}

\author{
A. B. Verchovsky, I. P. Wright and C. T. Pillinger \\ Planetary and Space Sciences Research Institute, The Open University, Walton Hall, \\ Milton Keynes, MK7 6AA, UK \\ Received 2003 May 2, accepted 2003 July 8
}

\begin{abstract}
A numerical model for ion implantation into spherical grains in free space has been developed. It can be applied to single grains or collections of grains with known grain-size distributions. Ion-scattering effects were taken into account using results of computer simulations. Possible isotope and element fractionation of the implanted species was investigated using this model. The astrophysical significance of the model lies in the possible identification of energetically different components (such as noble gases) implanted into presolar grains (such as diamond and $\mathrm{SiC}$ ) and in establishing implantation energies of the components.
\end{abstract}

Keywords: stars: circumstellar matter — stars: winds, outflows

\section{Introduction}

Laboratory investigations of presolar grains separated from meteorites over the last 15 years (Anders 1988; Anders \& Zinner 1993; Ott 1993; Zinner 1997; Hoppe \& Zinner 2000; Hoppe 2001; Ott 2001) have created the foundation for a new scientific direction - isotope astrophysics - that represents a coupling of detailed theoretical modelling of nucleosynthetic processes in stars with precise isotope measurements made in the laboratory. In some cases the agreement between measured and calculated isotopic compositions is so good that it leaves no doubts about the origin of the grains (Gallino, Busso, Picchio et al. 1990; Gallino, Busso \& Lugaro 1997). In many cases, however, it is only possible to deduce the class of astrophysical objects with which the grains might be connected (Amari \& Zinner 1997; Amari, Zinner, Jose et al. 2001). A general complication for most of the presolar materials is that they represent complex systems in terms of the stellar sources from which they originated and which are not easy to identify from analysis of collections of grains. In this sense isotope analyses of individual grains (micrometre dimensions or greater) is more informative, as has been done successfully in the case of SiC (Hoppe \& Ott 1997), graphite (Amari, Anders, Virag et al. 1990), and alumina (Nittler 1997) using the ion microprobe as an analytical tool. However small grains, such as presolar diamonds (the size of which is measured in 1000 s of atoms), cannot be analysed in this way, because of the limits imposed by counting statistics. Similarly minor constituents of larger grains (such as noble gases) are not measurable in individual samples. In such circumstances the only way to obtain any insight into the components or grains of interest is to analyse appropriate collections.

When analysing collections of grain, an additional relevant piece of information of use in interpreting the history of the samples is a knowledge of the incorporation mechanisms of minor components into the host. In this paper we will concentrate in particular on minor isotopically anomalous components of noble gases such as $\mathrm{Ne}$ and $\mathrm{Xe}$ implanted into nanometre-sized diamond grains. Such an approach can help with the identification of a number of independent events during which the components might have been acquired.

In simplistic terms there are two different mechanisms which might allow incorporation of trace materials into newly forming grains: they can either be trapped during grain growth directly from the gaseous feedstock, forming separate phases, solid solutions or structural defects, or they can subsequently be implanted some time after the grains have been formed. In general, the concentrations of components acquired during grain growth are expected to be independent of grain size; in principle however, under certain conditions, some systematic variation of concentration with grain size may be associated with, for example, variations in partial pressures of condensing species during grain growth. This results in complex dependencies of concentrations as a function of grain size, and is hardly predictable theoretically. In contrast, implantation is a strongly grain-size dependent process with a certain simple theoretical form. Thus, measurement of the distribution of minor components in grains (or collection of grains) of different sizes should allow a distinction between these two mechanisms.

From the data obtained so far it is clear that presolar grains of different types have acquired the bulk of their isotopically anomalous noble gas components through ion implantation. For example, the concentrations of noble gases in $\mathrm{SiC}$ grain-size fractions are clearly related to their grain size (Lewis, Amari \& Anders 1994). Recent analyses of grain-size fractions of presolar diamonds (Verchovsky, Fisenko, Semjonova et al. 1998) also show a strong grain-size dependence of noble gas concentrations. The only way that these relationships can be explained is through ion implantation into previously noble gas free grains.

To account for the variations, not only qualitatively but quantitatively as well, an implantation model applicable to presolar grains has been developed. 


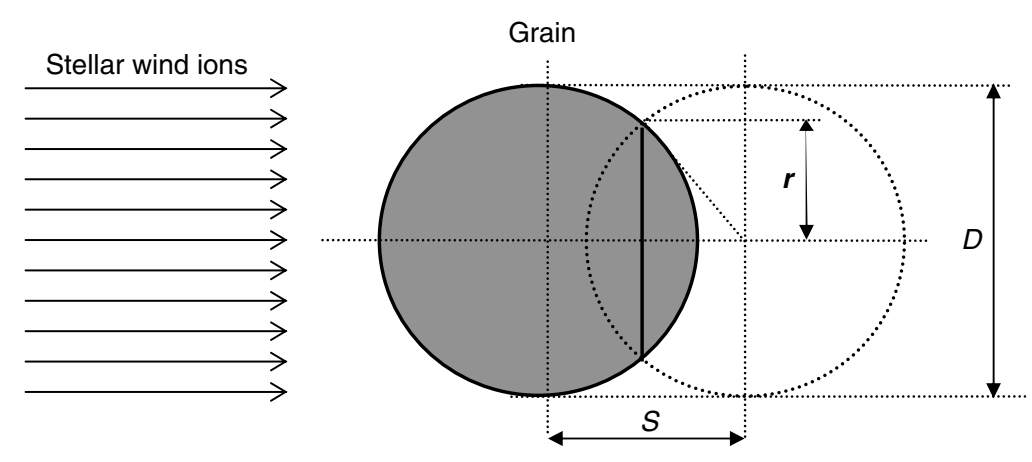

Figure 1 Illustration of the simple implantation model.

\section{Implantation Modelling}

\subsection{Single Grain Model}

As a starting point we consider here a simple case which can serve as a basic model for subsequent more detailed studies. In this preliminary approach we consider only the energy of the ions and not their overall mass, thus we do not distinguish between different elements such as $\mathrm{Ne}$ or Xe. In each case we consider the effect of ion implantation into a population of pre-existing grains in space - the exact formation mechanism of the grains is unimportant. Of necessity, we make some assumptions about the grains, for instance that they are defect-free and spherical in shape.

\subsubsection{Ions Accelerated by Stellar Wind or Shock Waves}

We assume that an ideally spherical grain with diameter $D$ is irradiated with ions of certain energy from one side in free space. We also assume that at the same energy the projected ranges $(S)$ of all ions in the grain are equal. In this case the cross-section of the grain for ion capture is equal to the area of a circle of radius $r$ formed by intersection of two spheres of diameter $D$, shifted from each other by the distance $S$ (Figure 1):

$$
\sigma=\pi \cdot r^{2}=\frac{\pi \cdot\left(D^{2}-S^{2}\right)}{4} .
$$

In our modelling the exact units of $D, S$ and $r$ are unimportant since we are ultimately only interested in dimensionless parameters such as the ratio of grain diameter to implantation range $(D / S)$. The concentration $\left(c\left[\mathrm{~cm}^{-3}\right]\right)$ of the implanted species as a function of ion fluence $\left(\Phi\left[\mathrm{cm}^{-2}\right]\right)$, grain diameter and implantation range is given by:

$$
c(D)=\Phi \cdot \frac{3}{2} \cdot \frac{D^{2}-S^{2}}{D^{3}} .
$$

The relationship is shown graphically in Figure 2a. As can be seen, the curve consists of two distinct parts. When the ratio of grain diameter to implantation range is $<1.8$ the concentration of the implanted component increases with grain size. This happens because only a fraction of the total ions stop within the grain; the rest traverse the grain without being captured. The concentration continues to increase with increasing grain size until almost all incoming ions stop within the grain. In this range of implantation depth the implanted ions reveal features of a volume-correlated component. For $D / S>1.8$ hypothetical concentrations decrease with increasing grain size, approaching a function of $1 / D$, which is a characteristic feature of a surface-correlated component. Because we have assumed a symmetrical sphere, the solution is valid for grain rotation as well as for the case where ions come equally from any direction. A useful parameter is the fraction of ions $(f)$ which stop inside the grain; this can be calculated in this case as the ratio of ion capture cross-section to the grain cross-section:

$$
f=\pi \cdot r^{2} /\left(\pi \cdot D^{2} / 4\right)=\frac{D^{2}-S^{2}}{D^{2}} .
$$

\subsubsection{Scattering Effects: TRIM Model}

We now consider additional effects, and in particular begin to constrain the modelling to specific noble gas ions (i.e. ${ }^{4} \mathrm{He}^{+},{ }^{22} \mathrm{Ne}^{+},{ }^{36} \mathrm{Ar}^{+},{ }^{84} \mathrm{Kr}^{+}$and ${ }^{132} \mathrm{Xe}^{+}$). The above assumes that no ion scattering occurs along the projected implantation direction. Such an assumption may be reasonable for light element targets bombarded with ions at implantation energies of a few MeV. However, at energies of a few hundred $\mathrm{keV}$ or less, both projected and lateral scattering of implanted ions becomes an important factor (Figure 3 ) that needs to be taken into account. In addition it is also necessary to consider the effects of backscattering, which is especially important for light ions like $\mathrm{He}^{+}$ and $\mathrm{Ne}^{+}$. To take account of these effects we use a TRIM approximation - a computer program which simulates ion implantation into solids using Monte Carlo method and developed by J. F. Ziegler (Ziegler 2003) — as follows:

1. For each noble gas ( $\mathrm{He}, \mathrm{Ne}, \mathrm{Ar}, \mathrm{Kr}$ and $\mathrm{Xe}$ ) we have made standard TRIM calculations for implantation into thick targets (diamond and $\mathrm{SiC}$ ) assuming a certain energy in each case. Standard calculations mean simulation of implantation when the incident ion beam encounters the target at a single point. As the TRIM code gives coordinates of all ions stopped within the target and the number of 

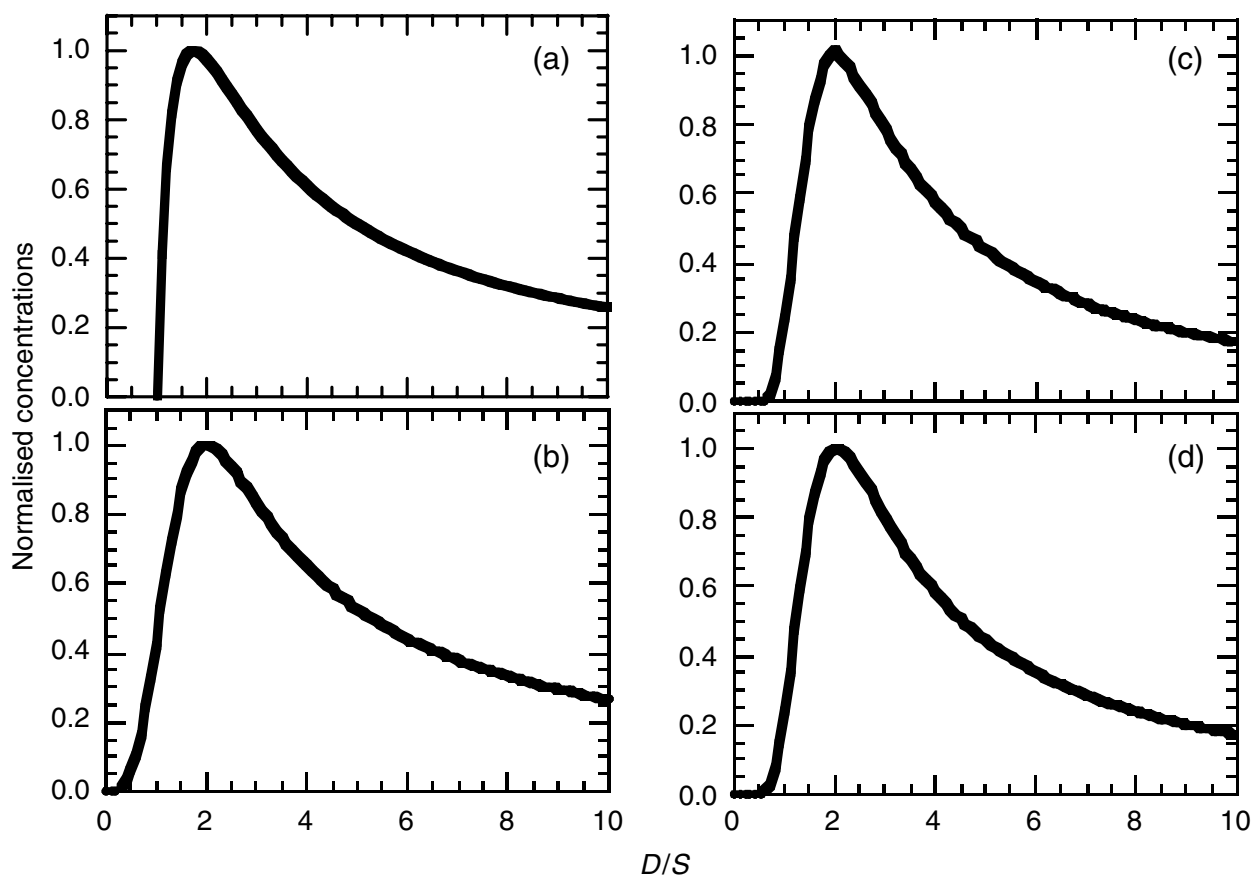

Figure 2 Grain size dependence of concentrations normalised to maximum values (the average implantation range in all cases is assumed to be 1): (a) single grain with constant implantation range; (b) TRIM model (He in diamond at $0.25 \mathrm{keV}$ ) for single grain; (c) TRIM model as in (b) for a collection of grains with normal grain size distribution; the standard deviation is assumed to be \pm 0.5 of the median value for all grain collections; (d) TRIM model as in (b) and (c) for a collection of grains with the same grain size distribution as in (c) and normal energy distribution (standard deviation is \pm 0.3 of the median value).

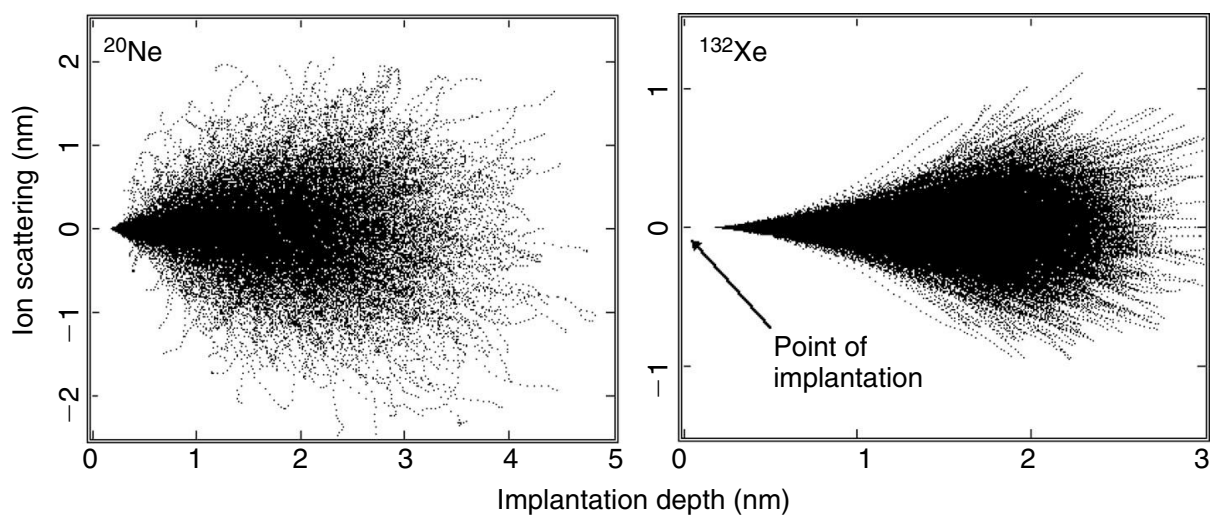

Figure 3 TRIM simulation of the implantation of ${ }^{20} \mathrm{Ne}$ and ${ }^{132} \mathrm{Xe}$ at $1.2 \mathrm{keV}$ into diamond target.

backscattering events, it is possible to calculate a fraction of total ion fluence stopped within a sphere of a given diameter. Simulations were run until 500010000 ions were stopped inside the targets. A separate computer program was written to calculate the ion fraction.

2. By changing the ion angle of incidence of the beam with respect to the surface of the hypothetical grain we were able to determine the dependence of ion implantation with angle. This was then smoothed using a cubic spline (Figure 4a). The integral of the smooth curve over the angle range from $0^{\circ}$ to $90^{\circ}$ gives a total fraction of ions stopped in a spherical grain ( $\mathrm{F}_{\mathrm{ISG}}$ ) which is valid for the model considered in $\$ 2.1 .1$.

3. By making calculations for spheres of different diameters the dependence of FISG $_{\text {ISG }}$ grain size was established (Figure 4b). The relationship was then fitted with the simple functions:

$$
\begin{aligned}
& Y=\frac{a \cdot X^{2}-b}{X^{2}+c} \text { or } \\
& Y=\frac{a \cdot X^{n}}{X^{n}+b},
\end{aligned}
$$

where $Y$ is the $\mathrm{F}_{\text {ISG }}$ and $X$ is the ratio of grain diameter to the average projected implantation range $(D / S)$, and $a, b, c$ and $n$ are constants dependent on the type of noble gas, implantation energy and nature of target (Table 1). Using an analogy with the basic model (§2.1.1) the effective cross-section of ions captured by a spherical grain can by found for the case as 

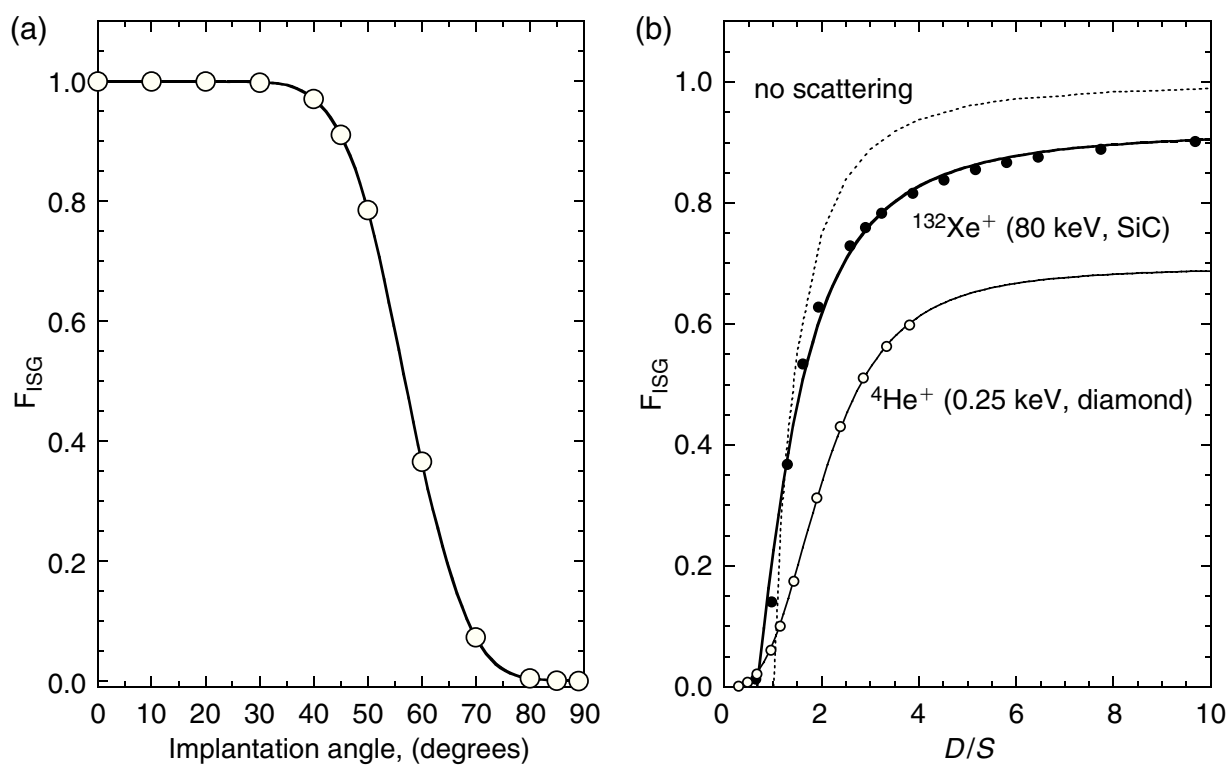

Figure 4 TRIM model calculations. (a) an example of the dependence of the incident angle on calculated fraction ( $\left.\mathrm{F}_{\text {ISG }}\right)$ of ${ }^{132} \mathrm{Xe}$ ions with energy $1.2 \mathrm{keV}$ which stop inside a hypothetical spherical diamond grain of $2 \mathrm{~nm}$ in diameter. (b) dependence of ion implantation efficiency, expressed as $\mathrm{F}_{\mathrm{ISG}}$, with the ratio of grain diameter $(D)$ to the average implantation range $(S)$. The line for the simple single grain model is expressed by $\mathrm{F}_{\text {ISG }}=\left((D / S)^{2}-1\right) /(D / S)^{2}$. The lines fitted to the TRIM model calculations for ${ }^{4} \mathrm{He}^{+}$implanted into diamond and ${ }^{132} \mathrm{Xe}^{+}$implanted into $\mathrm{SiC}$ are: $\left.\mathrm{F}_{\mathrm{ISG}}=0.694 \cdot(D / S)^{3}-1\right) /\left((D / S)^{3}+8.488\right)$ and $\mathrm{F}_{\mathrm{ISG}}=\left(0.921 \cdot\left((D / S)^{2}-0.412\right) /\left((D / S)^{2}+1.312\right)\right.$ respectively.

Table 1. Parameter of the functions $Y=\left(a \cdot X^{2}-b\right) /\left(X^{2}+c\right)$ and $Y=\left(a \cdot X^{n}\right) /\left(X^{n}+c\right)$ fitted by TRIM calculations for different noble gas isotopes, targets and implantation energies

\begin{tabular}{lllllllll}
\hline Ion & Target & $\begin{array}{l}\text { Energy } \\
(\mathrm{keV})\end{array}$ & $\begin{array}{l}\text { Average } \\
\text { range }(\mathrm{nm})\end{array}$ & $\begin{array}{l} \pm 3 \sigma \\
(\mathrm{nm})\end{array}$ & $a$ & $b$ & $c$ & $n$ \\
\hline${ }^{4} \mathrm{He}$ & Diamond & 0.25 & 2.10 & 2.0 & 0.694 & 8.488 & 0 & 3 \\
${ }^{4} \mathrm{He}$ & $\mathrm{SiC}$ & 100 & 500 & 300 & 0.842 & 5.270 & 0 & 4 \\
${ }^{20} \mathrm{Ne}$ & Diamond & 1.20 & 2.10 & 1.6 & 0.700 & 3.549 & 0 & 4 \\
${ }^{22} \mathrm{Ne}$ & Diamond & 1.20 & 2.13 & 1.6 & 0.710 & 3.561 & 0 & 4 \\
${ }^{22} \mathrm{Ne}$ & SiC & 500 & 650 & 300 & 0.785 & 5.195 & 0 & 5 \\
${ }^{36} \mathrm{Ar}$ & Diamond & 1.20 & 1.74 & 1.2 & 0.668 & 4.400 & 0 & 5 \\
${ }^{36} \mathrm{Ar}$ & $\mathrm{SiC}$ & 2000 & 1050 & 420 & 0.779 & 5.665 & 0 & 6 \\
${ }^{36} \mathrm{Ar}$ & $\mathrm{SiC}$ & 500 & 870 & 240 & 0.817 & 4.597 & 0 & 4 \\
${ }^{86} \mathrm{Kr}$ & Diamond & 1.20 & 1.87 & 1.0 & 0.623 & 4.417 & 0 & 7 \\
${ }^{84} \mathrm{Kr}$ & SiC & 2500 & 870 & 360 & 0.744 & 5.002 & 0 & 6 \\
${ }^{136} \mathrm{Xe}$ & Diamond & 1.20 & 2.06 & 0.8 & 0.607 & 5.443 & 0 & 8 \\
${ }^{132} \mathrm{Xe}$ & Diamond & 1.20 & 2.03 & 0.8 & 0.607 & 5.426 & 0 & 8 \\
${ }^{132} \mathrm{Xe}$ & SiC & 80 & 31 & 20 & 0.921 & 0.412 & 1.312 & 2 \\
\hline
\end{tabular}

$\left(\mathrm{F}_{\mathrm{ISG}} \cdot \pi \cdot D^{2}\right) / 4$ and the concentration is then given by:

$$
\begin{aligned}
& c(D)=\Phi \cdot \frac{3}{2} \cdot \frac{a \cdot D^{2}-b \cdot S^{2}}{D \cdot\left(D^{2}+c \cdot S^{2}\right)} \text { or } \\
& c(D)=\Phi \cdot \frac{3}{2} \cdot \frac{a \cdot D^{n-1}}{\left.D^{n}+b \cdot S^{n}\right)},
\end{aligned}
$$

and is shown in a graphical form in Figure 2b. As can be seen, the dependence reveals very similar features to those observed for the simple, single-grain model.

\subsection{Multiple Grain Model}

In practice, of course, we are not considering a single grain but rather a collection of grains. For the huge number of grains (that is statistically representative of the population) one needs to know the grain size distribution function $(F(D))$ in order to calculate the total concentration $(C)$ of an implanted species in the grain collection as a whole:

$$
C(D)=\int_{D_{0}}^{D} F(D) \cdot c(D) d D
$$

where $D_{0}-D$ is the range of grain sizes and $c(D)$ is the concentration in a single grain (eqn. 2).

In addition to the scattering effects on the target atoms at low energy (see \$2.1.3), variations in the projected ranges can also be caused by variations of the implantation energy. To take this effect into account we need 

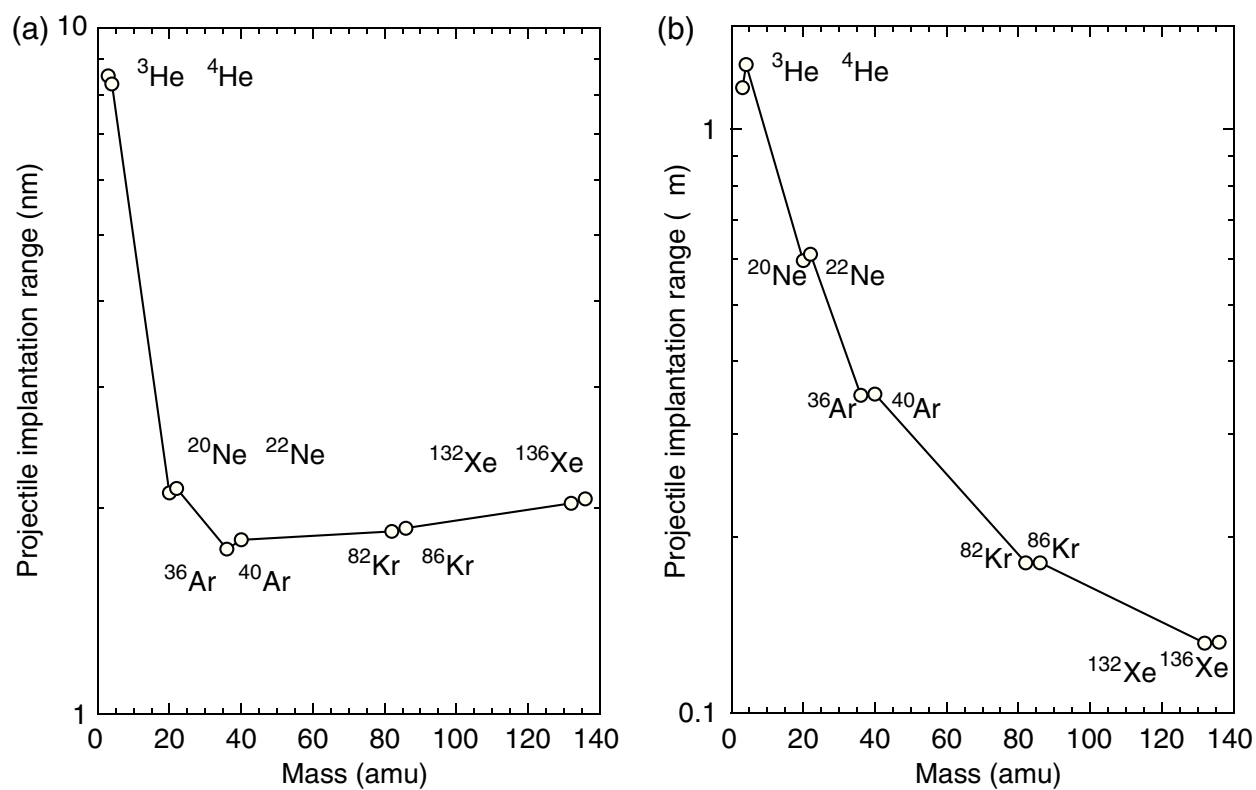

Figure 5 Ion implantation ranges for noble gas isotopes implanted into a diamond target at $1.2 \mathrm{keV}$ (a) and a SiC target at $500 \mathrm{keV}$ (b) calculated with the TRIM code.

to consider the distribution function of the implantation energies $(E(S))$ translated into variations of the projected ranges. Then the concentration is given by:

$$
C(D)=\int_{S_{0}}^{S} E(S) \int_{D_{0}}^{D} F(D) \cdot c(D) d D d S,
$$

where $S_{0}-S$ is the range of implantation depth.

Figure 2 shows a comparison between concentrations derived for a single grain (as in \$2.1) and those for collections of grains, assuming that the distribution function for $F(D)$ is given by the Gaussian function:

$$
F(D)=\frac{1}{\sigma_{D} \cdot \sqrt{2 \pi}} \cdot e^{\frac{-\left(D-D_{m}\right)^{2}}{2 \cdot\left(\sigma_{D}\right)^{2}}}
$$

and the distribution function for implantation energies is:

$$
E(S)=\frac{1}{\sigma_{S} \cdot \sqrt{2 \pi}} \cdot e^{\frac{-\left(S-S_{m}\right)^{2}}{2 \cdot\left(\sigma_{S}\right)^{2}}}
$$

As can be seen (Figures 2c and 2d) the dependence of concentration on grain size remains in general similar to that for the single grain model.

\subsection{Isotope and Element Variations during Implantation}

\subsubsection{Isotope Effects}

There are several isotope effects associated with implantation (e.g. Bernatowicz \& Hagee 1987). For instance, implantation at low energy causes reflection of ions from the surface and ejection of implanted ions by other impinging ions (sputtering effects). Furthermore, thermal diffusion can result in loss of implanted species. The processes are almost all mass-dependent and this will inevitably lead to a systematic isotope fractionation of implanted ions relative to their source reservoir. Such effects have been observed experimentally and in particular for noble gases (Bernatowicz et al. 1987; Ponganis, Graf \& Marti 1997; Koscheev, Gromov, Mohapatra et al. 2001). However, these isotope effects are not necessarily dependent on grain size and are therefore not particularly related to the model under discussion.

Of more importance here are any differences in implantation depths for different isotopic species (i.e. ions which otherwise are equivalent in energy, but differ in mass). Using the modelling technique described in the previous sections, isotope effects that may be experienced during the irradiation of grains in space can be investigated. First we consider the case of grain(s) being irradiated by a single species (e.g. Ne), existing in two (or more) different isotopic forms (e.g. ${ }^{20} \mathrm{Ne}^{+}$and ${ }^{22} \mathrm{Ne}^{+}$) with the same energy. Table 1 and Figure 5a show the results of TRIM calculations for noble gas isotopes implanted into diamond grains at relatively low $(1.2 \mathrm{keV})$ energies. Clearly, the heavier the mass of an isotope the larger its implantation range at the same energy (except for $\mathrm{He}$ where it is smaller for the heavier isotope), resulting in small but systematic differences for the parameters of the implantation model (Table 1). At higher energy (Figure 5b, implantation into $\mathrm{SiC}$ grains with $500 \mathrm{keV}$ ) similar differences in implantation ranges are observed only for isotopes of $\mathrm{He}$ and $\mathrm{Ne}$, heavier noble gases show practically no such difference at all. However in this case the differences in the implantation ranges between heavy elements ( $\mathrm{Ar}, \mathrm{Kr}$ and $\mathrm{Xe}$ ) are much more significant and opposite to those observed at the lower implantation energy (Figure 5a). Figure 6 shows the results of the model calculations for a number of grain-size fractions with normal grain size distributions characterised by a certain value of the standard deviation. The calculated isotope variations are significant for those 

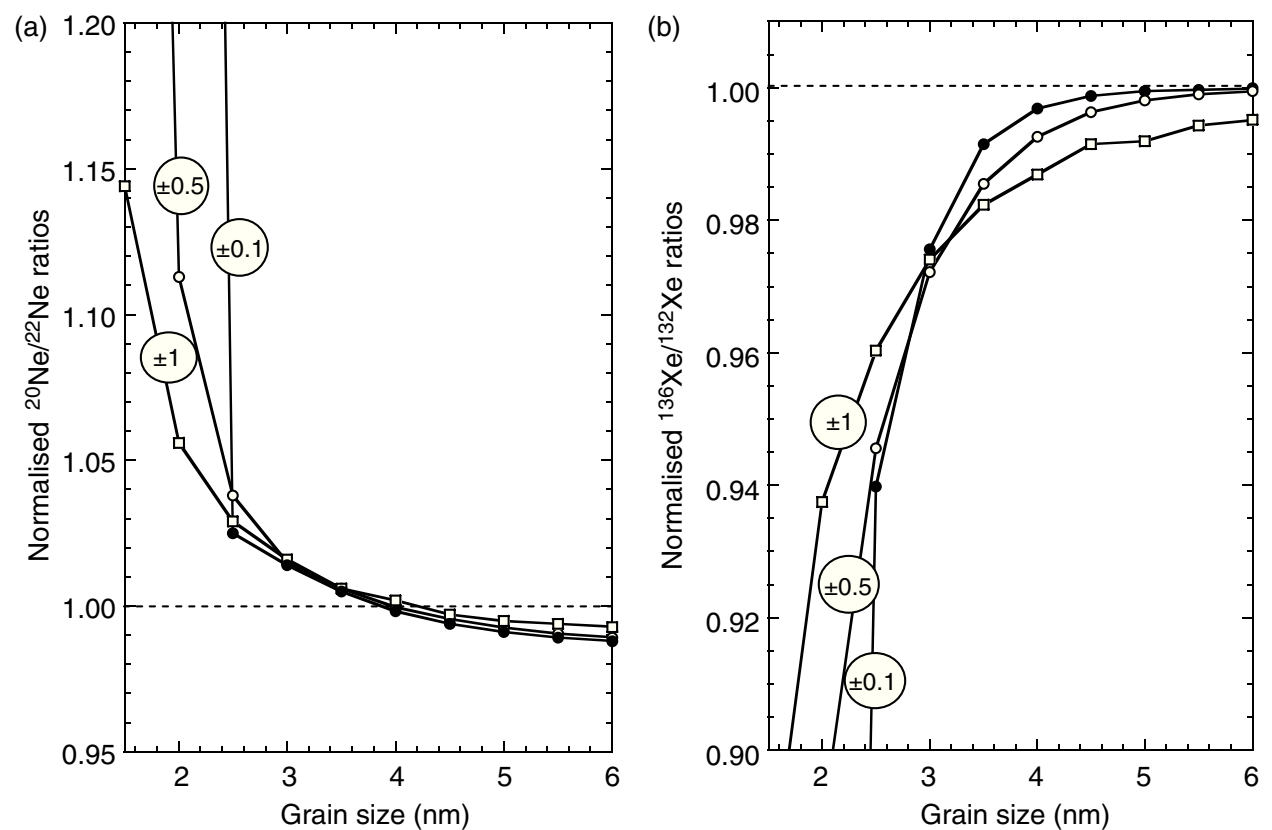

Figure 6 Isotope variations of implanted components at $1.2 \mathrm{keV}$ into diamond grains for (a) $\mathrm{Ne}$ and (b) Xe normalised to the original values before implantation. Each line represents the results of calculations made for a number of grain-size fractions with normal grain size distributions characterised by a particular $\sigma$ value. These values are shown as numbers in circles and represent a fraction of the median grain sizes. In this example we have plotted the effects versus mean grain size (in $\mathrm{nm}$ ) rather than the $D / S$ ratio to allow comparison with nanodiamonds from meteorites, which have grain sizes typically between 2 and $4 \mathrm{~nm}$. Parameters used for the calculations are taken from Table 1.

fractions that have grain sizes comparable to implantation ranges (i.e. low $D / S$ ratios). Furthermore, the magnitude of the observable effects are clearly related to the extent of grain size variations within an individual collection. It is thus obvious that the interpretation of data acquired from grains extracted from meteorites will be controlled by the ability to obtain tightly defined grain-size fractions.

These variations are comparable with those observed for noble gases in Efremovka diamond grain size fractions (Verchovsky et al. 1998) especially for Ne and, perhaps, for He. This means that the isotope effects associated with ion implantation of noble gases into presolar diamond should be taken into account in order to find the original noble gas isotopic compositions (i.e. before implantation). The same seems to be applied to meteoritic $\mathrm{SiC}$ grains to much less extent, since variations in the isotopic compositions of $\mathrm{He}$ and $\mathrm{Ne}$ between different $\mathrm{SiC}$ grain-size fractions (Lewis et al. 1994) are much larger, and the differences in the calculated implantation ranges for isotopes of these elements implanted at high energy (Figure 5b) are nearly the same as those for nanodiamonds.

Another related case is when isotopically different components of an individual species are ionised and implanted into the same target. To illustrate this we could imagine grains being irradiated by $\mathrm{Ne}$ from two different sources, one with ${ }^{20} \mathrm{Ne} /{ }^{22} \mathrm{Ne}$ ratio of $\mathrm{A}$ and energy $x$, and the other having ${ }^{20} \mathrm{Ne} /{ }^{22} \mathrm{Ne}$ ratio of $\mathrm{B}$ with energy $y(\neq x)$. The question to address is whether or not a population of such grains could merely exhibit $\mathrm{Ne}$ of the same homogeneous isotopic composition $\left({ }^{20} \mathrm{Ne} /{ }^{22} \mathrm{Ne}\right.$ somewhere between $\mathrm{A}$ and $\mathrm{B}$ depending on the relative abundance of the two components). In principle, depending upon implantation range, energy and size of individual grains, it is possible that ions of one particular energy will impart a volume-related signature, whilst those of another will be mainly surface-sited. In this case, grains of different size will record relative differences in the isotopic composition of the implanted species. Knowledge of this fact may help understand the irradiation record.

Figure 7 shows the result of a modelling exercise in which a collection of diamond grains is irradiated by two $\mathrm{Ne}$ components with two differnt energies. In all cases the relative abundance of the two components (as ions in space) is set to be $1: 1$ and the ${ }^{20} \mathrm{Ne} /{ }^{22} \mathrm{Ne}$ ratio for the mixture is defined to be 5 . As can be seen from Figure 7, the high energy ions (component 2) completely traverse the very smallest grains, leaving no signal. In contrast, low energy component 1 ions are trapped, resulting in the grains having an isotopic composition which reflects pure component 1 . As grain size increases there comes a point at which the higher energy component becomes implanted and the recorded isotope ratio starts to change systematically towards the value representing a mixing of the 2 components. Beyond a certain grain size the system is largely well mixed and there is no observed variation.

Quite similar isotope variations for noble gases are observed in presolar diamonds (Verchovsky et al. 1998) and $\mathrm{SiC}$ (Lewis et al. 1994) grain-size fractions, as well as for Ba (Zinner, Amari \& Lewis 1991; Prombo, Podosek, Amari et al. 1993) and Sr (Prombo, Podosek, Amari et al. 


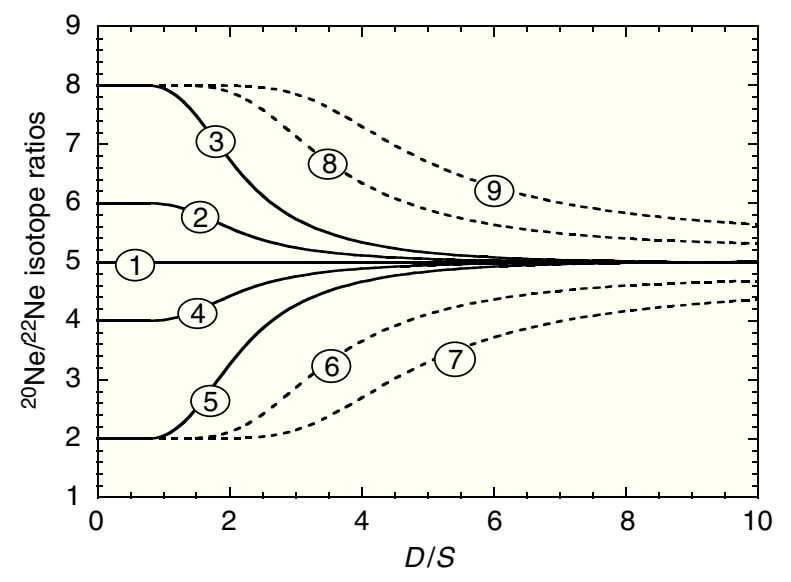

Figure 7 Isotope ratios of $\mathrm{Ne}$ as a function of grain size when two isotopically different components are implanted into the same grains (diamonds) with different energies. The solid lines represent a case of constant energies for each component (translated into $1 \mathrm{~nm}$ and $2 \mathrm{~nm}$ implantation ranges for low and high energies respectively) and variable isotopic compositions of the components provided the mixing ratio is $1: 1$ and the ${ }^{20} \mathrm{Ne} / 22 \mathrm{Ne}$ isotopic ratio of the mixture is always the same and equal to 5 . Lines 2, 3, 4 and 5 correspond to the following ${ }^{20} \mathrm{Ne} /{ }^{22} \mathrm{Ne}$ ratios for the low and high energy Ne: 6 and 4, 8 and 2, 4 and 6, 2 and 8 respectively. The dashed lines represent cases of implantation of two Ne components, in each case having different ${ }^{20} \mathrm{Ne} /{ }^{22} \mathrm{Ne}$ ratios and different energies. The ${ }^{20} \mathrm{Ne} /{ }^{22} \mathrm{Ne}$ ratios are 2 and 8 for lines 6 and 7, and 8 and 2 for lines 8 and 9 . For each case the two energies correspond to implantation ranges of 1 and $3 \mathrm{~nm}$ (lines 6 and 8 ) and 1 and $4 \mathrm{~nm}$ (lines 7 and 9). No isotope variations with grain size (line 1) would be observed when implantation energies of the $\mathrm{Ne}$ components are equal.

1992) in SiC grains. Thus, through measurements of the grain size and isotopic compositions of samples representing a collection of grains irradiated in space, it is possible to identify different implanted components provided their implantation energies are not identical, and also to calculate the implantation energies.

\subsubsection{Element Fractionation}

Larger variations are expected for elemental composition (compared to isotope variations) as a function of grain size. Indeed, at a given energy, different elements have significantly different implantation ranges as is shown for noble gases in Figure 5 and Table 1. Therefore, the elemental composition measured in different grain-size fractions is not equal to that in the gas mixture before implantation. The largest deviations from the original composition are expected in the fractions which have grain sizes comparable to the implantation ranges (Figure 8).

Even bigger effects are expected for shock accelerated ions, or a stellar wind generated by the mechanism similar to that producing solar wind, as in these cases ions have the same speed and their energies depend on their mass. In other words, a significant difference in the energies of individual elements is expected for the same component that eventually results in large elemental variations recorded in grains of different sizes with respect to the original composition.

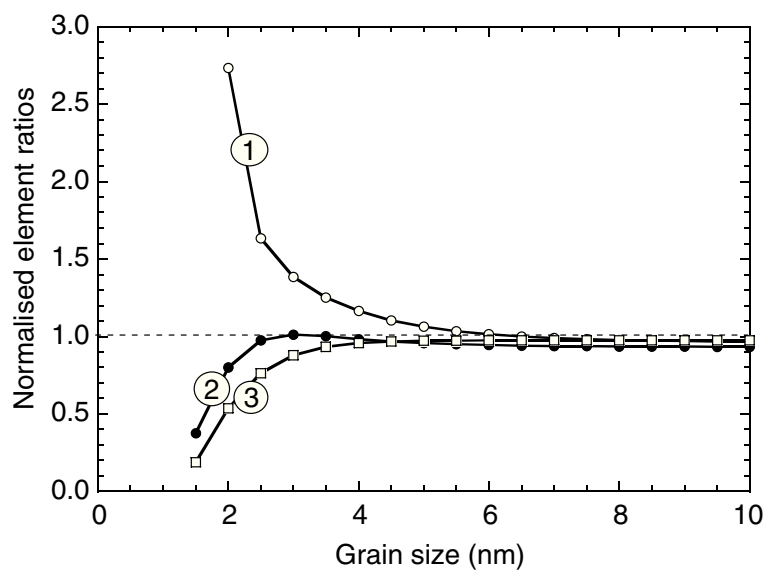

Figure 8 Elemental abundance variations as a function of grain size when a single component containing different noble gases is implanted into the same grains (diamonds) with the same energy $(1.2 \mathrm{keV})$ for every element. Each point corresponds to a grain-size fraction with a normal grain-size distribution and $\sigma$ value of 0.5 of the median grain size of the fraction. Lines 1, 2 and 3 represent ${ }^{36} \mathrm{Ar} /{ }^{20} \mathrm{Ne},{ }^{86} \mathrm{Kr} /{ }^{36} \mathrm{Ar}$ and ${ }^{132} \mathrm{Xe} /{ }^{86} \mathrm{Kr}$ elemental ratios respectively. Parameters for the calculations are taken from Table 1.

\section{Summary}

A theoretical model of ion implantation into grains in free space can be applied to individual grains or sizefractions of presolar grains extracted from meteorites. The model allows for recognition of isotopically different components implanted with different energies. Furthermore, it is possible to determine the energies for each of them. The model also indicates that isotopic and elemental compositions of implanted components recorded in the grains do not always correspond to those in their sources. The original compositions can however be found by analysis of grain-size fractions in combination with the model calculations. This is a valuable contribution to astrophysical information that can be used for detailed reconstruction of circumstellar conditions of presolar grain formation.

The application of the model for grain-size fractions of presolar diamonds and $\mathrm{SiC}$ will be published elsewhere (Verchovsky et al. 2003).

\section{References}

Amari, S., Anders, E., Virag, A., \& Zinner, E. 1990, Nature, 345, 238 Amari, S., \& Zinner, E. 1997, in AIP Conf. Proc, 402, Astrophysical Implications of the Laboratory Study of Presolar Materials, ed. E. K. Zinner (Woodbury, New York: American Institute of Physics), 287

Amari, S., Zinner, E., Jose, J., \& Hernanz, M. 2001, Nuclear Phys., 688,3

Anders, E. 1988, in Meteorites and the Early Solar System, ed. J. F. Kerridge \& M. S. Matthews (Tucson, AZ: Univ. Ariz, Press), 927

Anders, E., \& Zinner, E. 1993, Meteoritics, 28, 490

Bernatowicz, T. J., \& Hagee, B. E. 1987, Geochim. Cosmochim. Acta, 51, 1599

Gallino, R., Busso, M., \& Lugaro, M. 1997, in AIP Conf. Proc. 402, Astrophysical Implications of the Laboratory Study of Presolar Materials, ed. T. J. Bernatowicz \& E. K. Zinner (Woodbury, New York: American Institute of Physics), 115 
Gallino, R., Busso, M., Picchio, G., \& Renzini, A. 1990, Nature, 348,298

Hoppe, P. 2001, Nuclear Phys., 688, 94c

Hoppe, P., \& Ott, U. 1997, in AIP Conf. Proc. 402, Astrophysical Implications of the Laboratory Study of Presolar Materials, ed. T. J. Bernatowicz \& E. K. Zinner (Woodbury, New York: American Institute of Physics), 27

Hoppe, P., \& Zinner, E. 2000, J. Geophys. Res., 105, 10

Koscheev, A. P., Gromov, M. D., Mohapatra, R. K., \& Ott, U. 2001, Nature, 412, 615

Lewis, R. S., Amari, S., \& Anders, E. 1994, Geochim. Cosmochim. Acta, 58, 471

Nittler, L. R. 1997, in AIP Conf. Proc. 402, Astrophysical Implications of the Laboratory Study of Presolar Materials, ed. T. J. Bernatowitz \& E. K. Zinner (Woodbury, New York: American Institute of Physics), 59
Ott, U. 1993, Nature, 364, 25

Ott, U. 2001, Planet. Space Sci., 49, 763

Ponganis, K. V., Graf, T., \& Marti, K. 1997, J. Geophys. Res. E, Planets, 102, 19

Prombo, C. A., Podosek, F. A., Amari, S., \& Lewis, R. S. 1992, Lunar Planet. Sci. Conf., 23, 1111

Prombo, C. A., Podosek, F. A., Amari, S., \& Lewis, R. S. 1993, ApJ, 410,393

Verchovsky, A. B., Fisenko, A. V., Semjonova, L. F., Wright, I. P., Lee, M. R., \& Pillinger, C. T. 1998, Science, 281, 1165

Verchovsky, A. B., Wright I. P., \& Pillinger C. T. 2003, ApJ, in press

Ziegler, J. F. 2003, Particle interaction with matter, www.srim.org Zinner, E. 1998, Ann. Rev. Earth and Planet. Sci., 26, 147

Zinner, E., Amari, S., \& Lewis, R. S. 1991, ApJ, 382, L47 